\title{
Method to Distinguish between Space-Charge and Dipolar Relaxation in the TSDC Spectra of Polyethylene Electrical Insulation
}

\author{
J. Òrrit, J.C. Cañadas, J. Sellarès and J. Belana \\ Dept. de Física i Enginyeria Nuclear \\ Universitat Politècnica de Catalunya \\ c. Colom 11, Terrassa, E-08222 Spain \\ e-mail: jordi.orrit@upc.edu.
}

\begin{abstract}
Medium-voltage cross-linked polyethylene (MVXLPE) cables have an important role in the electrical power distribution system. For this reason, the study of XLPE insulation may lead to improve cable features and lifetime. Although relaxational analysis yield a lot of information about XLPE properties, sometimes their results are difficult to interpret. To overcome this handicap, we have used a combination of thermally stimulated depolarization currents (TSDC) and isothermal depolarization currents (IDC) techniques. In order to discard spurious effects from the semiconductor interfaces, preliminary measurements have been done on specially prepared cables. TSDC have been performed using conventional poling between $140{ }^{\circ} \mathrm{C}$ and $40{ }^{\circ} \mathrm{C}$. IDC measurements also have been carried out at temperatures between $90{ }^{\circ} \mathrm{C}$ and $110{ }^{\circ} \mathrm{C}$ in $2{ }^{\circ} \mathrm{C}$ steps. The TSDC spectra are dominated by a broad peak of uncertain origin. On the other hand, IDC show a combination of power and exponential currents. Exponential currents are fitted to a KWW model. The relaxation times obtained from the model present an Arrhenius behavior with $E_{a}=1.32 \mathrm{eV}$ and $\tau_{0}=3.29 \times 10^{-16} \mathbf{s}$. The KWW parameter obtained is $\beta=0.8$. The calculated depolarization current given by the exponential relaxation matches the predominant peak of TSDC spectra. Therefore, we conclude that in the MV cables studied the most visible peak of the TSDC spectrum has a dipolar origin.
\end{abstract}

Keywords-XLPE, TSDC, isothermal relaxation.

\section{INTRODUCTION}

A LTHOUGH nowadays Medium-voltage cross-linked polyethylene (MV-XLPE) cables are regarded as a commodity, some important points that affect their lifetime are not yet fully understood. Buildup of space charge in the bulk of the cable is known to be a major cause of failure [1]. For this reason, the study of space charge in MV-XLPE cables may provide clues about the most suitable composition (reticulants, antioxidants, ...) or manufacturing process (vulcanization time, ...) in order to enhance its operational lifetime [2]. Relaxation, especially space charge relaxation, is a very relevant subject [3], not only because of the role played by relaxational processes by themselves but also for the great amount of information about the electrical properties of the material that can be obtained from a relaxational study.

Among the techniques employed to study relaxations, thermally stimulated depolarization currents (TSDC) [4] stands out because of its high resolution and low equivalent frequency 978-1-4244-7944-3/10/\$26.00 C2010 IEEE

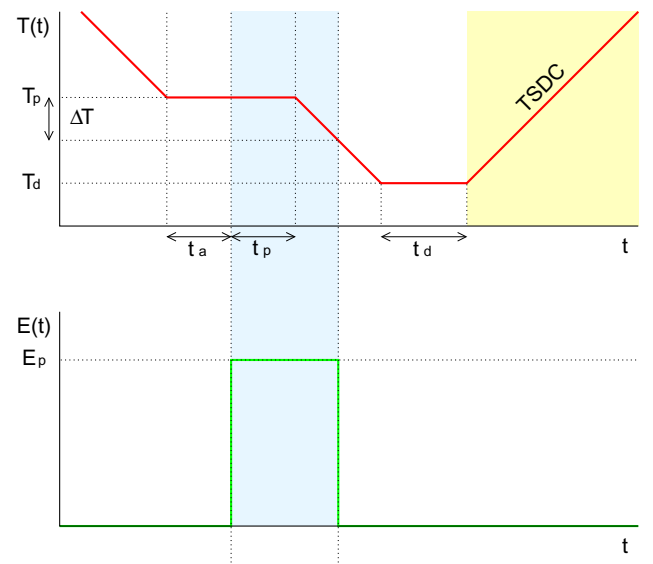

Figure 1. Representation of a TSDC experiment: annealing time $\left(t_{a}\right)$, poling time $\left(t_{p}\right)$, deposit time $\left(t_{d}\right)$, poling temperature $\left(T_{p}\right)$, poling temperature range $(\Delta T)$, deposit temperature $\left(T_{d}\right)$, poling feld $\left(E_{p}\right)$.

[5]. A diagram that shows how a TSDC experiment is performed is presented in f gure 1 .

A TSDC experiment begins with a combination of electrical poling and thermal treatment so one or more relaxational mechanisms are activated in a metastable way [6]. Then, the sample is heated at a constant rate to force the relaxation of these mechanisms. Its relaxation gives rise to a displacement current that is recorded together with the temperature of the sample. This thermogram is the so-called TSDC spectrum and ref ects the relaxations undergone by the sample.

TSDC has been employed successfully to f nd consistent differences in TSDC spectra of cable samples manufactured in the same factory under different conditions [7].

TSDC is particularly useful to study overlapping relaxations since its high resolution allows to distinguish between relaxations with close relaxation times. This is what happens in the case of XLPE, where a large number of relaxations can be identif ed in the TSDC spectrum [7]. Unfortunately, some problems arise during the study of XLPE cables by TSDC that make it diff cult to analyze the resulting spectra.

In frst place, TSDC spectra are strongly dependent on thermal history. This is a well-known characteristic of XLPE 
cables [7]. The effects of thermal history can be minimized performing each experiment on its own as-received sample.

Another problem is that cables are unusually thick compared with more commonly used samples and, therefore, only relatively low electric f elds can be employed to polarize them. The space-charge or dipolar character of a relaxation can be determined studying how the poling of the sample is related to the polarizing feld [8] but in the low electric f eld limit space charge and dipolar relaxations behave in a similar way. As a consequence, in this case this kind of analysis is not able to give conclusive results.

A way to overcome this diff culty is to employ a complementary technique to obtain information that can lead to the identif cation of one or several peaks. In this work we consider isothermal depolarization currents (IDC) as a complementary technique. There are several reasons that make this approach compelling. In $\mathrm{f}$ rst place, both techniques measure essentially the same physical effect, displacement current, so correlation between data obtained using both techniques is straightforward [9].

Moreover, contributions from dipoles or space charge are much easier to distinguish in IDC data than in a TSDC spectrum [10], especially if they are presented in a log-log plot.

The current may obey a power law [10]. Among the possible causes of a power law discharge current are dipole depolarization, Maxwell-Wagner relaxation, electrode polarization or recombination of trapped space charge [11].

When there is no probability of retrapping, the recombination process of space charge leads to an exponential current [12], that has only an horizontal asymptote for short times. This type of current can also be obtained by depolarization of molecular dipoles. In fact, recombination without retrapping happens when the displacement of space charge during poling has been so small that trapped charges act like small dipoles, sometimes called Gerson dipoles [13].

Therefore, we will attribute exponential current to dipolar relaxation, including under this denomination depolarization of molecular dipoles and recombination of Gerson dipoles.

In section III-A we will give the expressions that follow these currents and discuss its range of validity.

Combining TSDC and IDC we expect to conf rm previous assumptions about relaxations found in the TSDC spectrum of MV-XLPE cables [7] and to demonstrate how the correlation of TSDC with IDC data can improve our understanding of relaxation mechanisms in situations where the usual analysis of TSDC data is diff cult, or even impossible, to carry out.

\section{EXPERIMENTAL}

The experimental setup for IDC consists of a Kottermann 2715 forced air oven controlled by a Eurotherm 818P PID temperature programmer. Inside, two identical cable samples were placed close together in parallel position. One of them is used to host the temperature probe inside the insulating layer and IDC measurements were performed on the other one. The cable core and the outer electrode were connected to a Brandenburg $807 \mathrm{R}(3-30 \mathrm{kV})$ potential source and to a Keithley

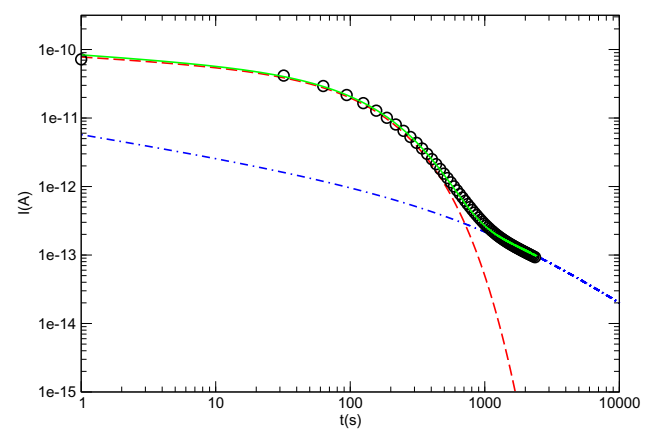

Figure 2. (o) IDC curve for $T_{p}=104{ }^{\circ} \mathrm{C}, t_{p}=1800 \mathrm{~s}$ and $V_{p}=10 \mathrm{kV}$ in a $\log -\log$ diagram; $(-)$ theoretical $\mathrm{ft} ;(--) \mathrm{KWW}$ component; $(\cdot-\cdot)$ power law component.

6514 electrometer, respectively. The TSDC experimental setup is similar to the IDC one and has been described in previous works [7].

Cable samples were provided by General Cable. They consist of a cylindrical insulating XLPE layer with $7 \mathrm{~mm}$ and $13 \mathrm{~mm}$ of inner and outer radii, respectively. The samples had $0.5 \mathrm{~mm}$ thick semiconducting layers in contact with the inner and external surfaces of XLPE. The insulating material was crosslinked mixing low density PE with a crosslinking agent (di-tert-butyl peroxide) and heating it up to a temperature higher than $200{ }^{\circ} \mathrm{C}$ to produce a vulcanization reaction.

The samples were cut from cable into $18 \mathrm{~cm}$ long sections. The semiconductor layers were used as electrodes. To avoid short circuits, the external layer was partially removed from the sample ends, leaving an $8 \mathrm{~cm}$ wide semiconductor strip centered in the sample. The cable core of twisted aluminum wires was used to make contact with the inner semiconductor layer. The contact with the outer semiconductor layer was made with an adjustable metallic clamp.

IDC measurements were performed at several temperatures $\left(T_{p}\right)$ between 90 and $110^{\circ} \mathrm{C}$ in $2{ }^{\circ} \mathrm{C}$ steps. The poling time $\left(t_{p}\right)$ was $1800 \mathrm{~s}$.

TSDC spectra were obtained by cooling down from $140{ }^{\circ} \mathrm{C}$ $\left(T_{p}\right)$ with a $12 \mathrm{kV}$ applied voltage until $40^{\circ} \mathrm{C}\left(\Delta T=100^{\circ} \mathrm{C}\right)$, and, after $5 \mathrm{~min}\left(t_{d}\right)$, heating up to $140^{\circ} \mathrm{C}$ again. Samples were polarized without an isothermal polarizing stage so $t_{a}=t_{p}=$ 0 . The heating or cooling rate of all the ramps was $2{ }^{\circ} \mathrm{C} / \mathrm{min}$.

Figure 2 presents an example of IDC experimental data. The power law $\mathrm{ft}$ was very unsatisfactory and therefore it was necessary to consider other possibilities. Adding an exponential term to the power term was revealed as the best way to overcome this problem, as seen in f gure 2 . The details will be given in the next section. However, to ensure that the effect that was measured had its origin on the dielectric properties of the cable insulation several previous experiments were performed.

In these experiments it was found that the exponential current was fainter on fat $2 \mathrm{~mm}$ thick samples and could not be detected on very thin $16 \mu \mathrm{m}$ thick samples. Other factors, such as shape or presence of semiconductor layers, had no effect on the current. 


\section{RESUlTS AND DiscUSSION}

\section{A. General equations}

In its origin the Kohlrausch-Williams-Watts (KWW) model was introduced as an empirical improvement to existing models. Nowadays it is interpreted as a way to take into account a distribution of relaxation times [14].

The KWW displacement current density is

$$
J=P_{0} \beta z^{\beta-1} \frac{\exp \left(-z^{\beta}\right)}{\tau}
$$

whew the reduced time $(z(t))$ is given by

$$
z(t) \equiv \int_{0}^{t} \frac{d t}{\tau}
$$

We can now apply equation 1 to two interesting cases: isothermal depolarization currents (IDC) and thermally stimulated depolarization currents (TSDC). In the f rst case we can safely assume that $\tau$ does not change with time, since this is the behavior predicted by the Arrhenius or the WLF models. This leads to a great simplif cation of equation 2 for the IDC case

$$
z(t)=\frac{t}{\tau}
$$

Substituting in equation 1 we obtain

$$
J(t)=P_{0} \beta \frac{t^{\beta-1}}{\tau^{\beta}} e^{-\left(\frac{t}{\tau}\right)^{\beta}}
$$

This is the stretched exponential current, or, simply, exponential current. In fact, we can expect that the IDC has also a free charge component that we denote as power current [11]

$$
J(t)=C t^{\alpha}\left[1-\left(\frac{1+t_{p}}{t}\right)^{\alpha}\right]
$$

where $\alpha<0$. Therefore we will $\mathrm{ft}$ the IDC to

$$
J(t)=C t^{\alpha}\left[1-\left(\frac{1+t_{p}}{t}\right)^{\alpha}\right]+D t^{\beta-1} e^{-\left(\frac{t}{\tau}\right)^{\beta}} .
$$

Since intensity is proportional to density current, through the area of the electrode, we can $\mathrm{ft}$ the intensity curves using this expression.

The second case is somewhat more complicated because depolarization takes place during a heating ramp. Assuming a constant heating rate $v$, temperature will be given by

$$
T(t)=T_{d}+v t \text {. }
$$

Since it is usual to plot $J$ in terms of $T$, we express $z$ as a function of temperature

$$
z(T)=\frac{1}{v} \int_{T_{d}}^{T} \frac{d T}{\tau(T)}
$$

so we can compare the experimental plot to [15]

$$
J(T)=P_{0} \exp \left\{-z^{\beta}(T)+\ln \left[\frac{\beta}{\tau(T)} z^{\beta-1}(T)\right]\right\} .
$$

As in the previous case $I(T) \propto J(T)$ and the expression can be applied to intensity curves.

\section{B. Data analysis}

It is very diff cult to establish the exact nature of the power current. Nevertheless it does not matter for the end of this work, because we are focused only on currents that can cause TSDC peaks, and this is not the case for the power current.

The exponential relaxation shows the typical behavior of a thermally activated process. For lower temperatures it shows up later. As a consequence, at low temperatures the power current determines the IDC response for short times while the exponential current is the responsible of the larger time response. Instead, at higher temperatures the opposite interplay between currents occurs.

The numeric result of all the $\mathrm{f}$ ts is presented in table I. The values of $C$ tend to diminish with increasing temperatures while $D$ remains more or less constant. The parameter $\alpha$ that characterizes the power current changes as a consequence of structural change, most probably the fusion of the material. Instead, the parameter $\beta$ does not change signif cantly throughout all the experiments. This can be interpreted as a sign that the exponential current is not related to XLPE itself but to some other component incorporated at the manufacturing process. Anyway, we will assume that $\beta$ does not depend on temperature and we will take it as a characteristic parameter of the relaxation.

TABLE I

FITTING RESULTS.

$\begin{array}{cccccc}T & C(\mathrm{~A}) & \alpha & \beta & D(\mathrm{~A}) & \tau(\mathrm{s}) \\ 90 & 1.01 \times 10^{-10} & -1.29 & 0.82 & 8.66 \times 10^{-11} & 7.59 \times 10^{2} \\ 92 & 4.12 \times 10^{-11} & -0.09 & 0.77 & 2.02 \times 10^{-10} & 5.29 \times 10^{2} \\ 94 & 1.56 \times 10^{-11} & -0.15 & 0.84 & 1.71 \times 10^{-10} & 3.37 \times 10^{2} \\ 96 & 2.35 \times 10^{-11} & -0.12 & 0.77 & 1.95 \times 10^{-10} & 3.10 \times 10^{2} \\ 98 & 6.33 \times 10^{-12} & -0.13 & 0.78 & 2.11 \times 10^{-10} & 3.07 \times 10^{2} \\ 100 & 1.43 \times 10^{-11} & -0.30 & 0.79 & 2.78 \times 10^{-10} & 2.81 \times 10^{2} \\ 102 & 8.98 \times 10^{-12} & -0.14 & 0.80 & 2.87 \times 10^{-10} & 1.84 \times 10^{2} \\ 104 & 6.41 \times 10^{-12} & -0.30 & 0.88 & 7.90 \times 10^{-11} & 1.19 \times 10^{2} \\ 106 & 2.46 \times 10^{-11} & -0.41 & 0.76 & 2.12 \times 10^{-10} & 1.12 \times 10^{2} \\ 108 & 3.20 \times 10^{-12} & -0.48 & 0.83 & 2.90 \times 10^{-10} & 1.02 \times 10^{2} \\ 110 & 3.56 \times 10^{-12} & -0.46 & 0.87 & 2.55 \times 10^{-10} & 7.01 \times 10^{1}\end{array}$

We can assume that the behavior of the $\tau$ parameter in table I follows Arrhenius law

$$
\tau=\tau_{0} \exp \left(\frac{E_{a}}{k T}\right)
$$

as it can be seen in f gure 3 . The linear regression plotted in this fgure reads $\ln (\tau)=-35.7+1.53 \times 10^{4} / T$, this is, $\tau_{0}=3.29 \times 10^{-16} \mathrm{~s}$ and $E_{a}=1.32 \mathrm{eV}$.

\section{Discussion}

Equation 9 can be employed to predict the shape of the TSDC current in terms of the obtained parameters. Of course, we will obtain just one of the many peaks present in the TSDC spectrum but if we compare the calculated current with the experimental spectrum we can identify the TSDC peak that corresponds to the exponential current found by IDC.

This comparison can be seen in f gure 4 . We can see that the predicted KWW peak $\mathrm{fts}$ rather well to the main peak of the TSDC spectra, placed at $368{ }^{\circ} \mathrm{C}$. A $3 \mathrm{~K}$ shift towards 


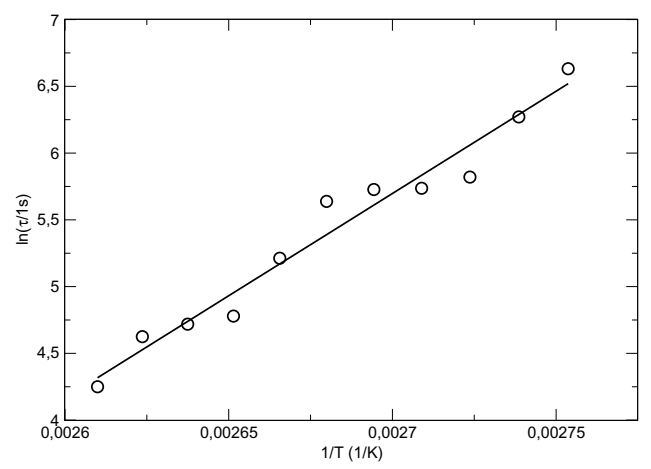

Figure 3. Arrhenius plot for relaxation times $(\tau)$ versus $T^{-1}$.

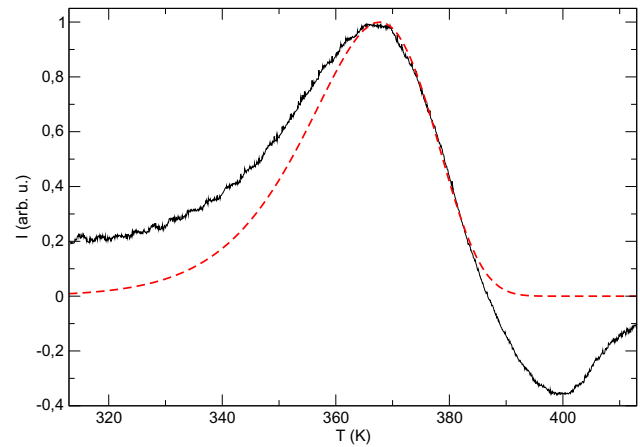

Figure 4. (-) TSDC curve for $T_{p}=140{ }^{\circ} \mathrm{C}, T_{d}=40{ }^{\circ} \mathrm{C}, t_{d}=5 \mathrm{~min}$ and $v=2{ }^{\circ} \mathrm{C} / \mathrm{min} ;(--)$ Simulated TSDC from equation 9 and for $E_{a}=$ $1.32 \mathrm{eV}, \tau_{0}=3.29 \times 10^{-16} \mathrm{~s}$ and $\beta=0.8$.

lower temperatures has been applied to the predicted curve to obtain a better concordance. This difference can be due to a temperature gradient inside the oven or, simply, to uncertainty in the $\mathrm{ft}$ results. As usual in most relaxation models, the KWW model tends to underestimate the amount of current before the maximum of the peak. Other than these two disparities, the agreement between both peaks is noticeable.

Taking this into account, we state that the IDC exponential current and the $95^{\circ} \mathrm{C}$ TSDC peak are due to the same physical cause. We will discuss in the following lines which could be that cause.

The most probable cause of the exponential current is polarization of molecular dipoles in the cable bulk. We have seen that usually dipolar currents adopt an exponential form.

The shape of the current is also compatible with recombination of Gerson dipoles but probably any trapped space-charge would give rise to a more irregular peak [16]. Nevertheless this possibility can not be completely discarded.

Since polyethylene is not a polar material, dipoles would come from additives introduced during the manufacturing process, either directly or from by-products. The fact that the exponential current does not show up on small samples, with a larger surface-to-volume ratio, would suggest a surface origin for the power current, for example electrode polarization, a very common process.

\section{CONCLUSIONS}

In a previous work about the TSDC spectrum of MV XLPE cables, it was found that the large heteropolar peak placed before the homopolar peak presented a dipole-like behavior [7]. The main evidence was that polarization turned out to be proportional to the applied feld, as it usually happens with dipoles. Anyway, space charge polarization also tends to be proportional to the applied feld for low f eld values. Measurements had to be performed on full cable samples and, therefore, even high poling potentials lead to weak poling f elds.

For this reason it was interesting to seek conf rmation for these fndings using another technique. IDC was a suitable technique because the shape of the current can be used to identify its cause. With this technique, an exponential current has been found that corresponds to the aforementioned peak. Thus, the dipolar character of the peak is confrmed by a complementary technique.

The peak could be caused by molecular dipoles, that would come from additives introduced during the manufacturing process, of from microscopic displacement of space charge. There are some indications that make more likely the frst option, although the second one can not be discarded.

These experiments are just one more step towards the goal of elucidating if the degradation processes that lead to cable failure can also be monitored with this technique. Further research on this subject could lead to interpretation of the spectra that would allow to assess the state of the cables in a non-destructive way [2].

\section{ACKNOWLEDGEMENTS}

This work has been partially f nanced by AGAUR, Generalitat de Catalunya (2009 SGR 01168). The authors thank General Cable for providing cable samples.

\section{REFERENCES}

[1] Y. Zhang, J. Lewiner, C. Alquie and N. Hampton, IEEE Trans. DEI 3, 778-783 (1996).

[2] I. Tamayo, J. Orrit and J. A. Diego, in "Proceedings of Jicable'07, ParisVersailles, France", p. C716, SEE (2007).

[3] M. Mudarra and J. Belana, Polymer 38, 5815-5821 (1997).

[4] R. Chen and Y. Kirsh, Analysis of thermally stimulated processes, chapter 3, pp. 60-81, Pergamon, Oxford, 1st edition (1981).

[5] J. Menegotto, P. Demont, A. Bernes and C. Lacabanne, J. Pol. Sci. Part B: Pol. Phys. 37, 3494-3503 (1999).

[6] M. Zielinski and M. Kryszewski, Phys. Stat. Sol. A 42, 305-314 (1977).

[7] I. Tamayo, J. Belana, J. C. Cañadas, M. Mudarra, J. A. Diego and J. Sellarès, J. Pol. Sci. Part B: Pol. Phys. 41, 1412-1421 (2003).

[8] J. Belana, M. Mudarra, J. Calaf, J. C. Cañadas and E. Menéndez, IEEE Trans. EI 28, 287-293 (1993)

[9] J. Ménégotto, P. Demont and C. Lacabanne, Polymer 42, 4375-4383 (2001).

[10] D. K. Das-Gupta, IEEE Trans. DEI 4, 149-156 (1997).

[11] V. Adamec and J. H. Calderwood, J. Phys. D: Appl. Phys. 11, 781-800 (1978).

[12] J. T. Randall and M. H. F. Wilkins, Proc. R. Soc. Lond. A 184, 365-389 (1945).

[13] R. Gerson and J. H. Rohrbaugh, J. Chem. Phys. 23, 2381-2388 (1955).

[14] F. Álvarez, A. Alegría and J. Colmenero, Phys. Rev. B 47, 125-130 (1993).

[15] A. Alegría, L. Goitiandía and J. Colmenero, Polymer 37, 2915-2923 (1996).

[16] J. A. Diego, J. Belana, J. Òrrit, J. Sellarès, M. Mudarra and J. C. Cañadas, J. Phys. D: Appl. Phys. 39, 1932-1938 (2006). 